\title{
Auslander Reiten Quiver of Nakayama Algebra type Dynkin Graph $A_{n}$
}

\author{
Faisal, Irawati \& Intan Muchtadi-Alamsyah \\ Algebra Research Group \\ Faculty of Mathematics and Natural Sciences, Institut Teknologi Bandung, \\ Jalan Ganesha 10 Bandung 40132, Indonesia \\ Email: ntan@math.itb.ac.id
}

\begin{abstract}
In this paper we will determine Auslander Reiten quiver of Nakayama algebra with quiver type Dynkin graph $A_{n}$ for all natural number $\mathrm{n} \geq 2$. The ARquiver is a visualization of module category of finite dimensional algebras. From the AR-quiver of an algebra $A$ we may know all the isomorphism classes of indecomposable modules in mod A and the homomorphism between them. Once we get the general shape of the AR-quiver of this algebra, we will use it to compute a tilting module of this algebra.
\end{abstract}

Keywords: AR-quiver; Nakayama algebra; quiver; Dynkin type A.

\section{$1 \quad$ Introduction}

Representation theory of finite dimensional algebras provides a way to describe an algebra and a module over an algebra using directed graph or quiver. Not only for the algebra and module, the module category of an algebra can also be represented by quiver. The way to do this is by using the theory of almost split sequences and irreducible morphisms. These were introduced by Auslander [1,2] and Auslander and Reiten [3]. Quiver representation of this module category called Auslander-Reiten quiver or simply AR-quiver. In this paper we will determine the AR-quiver of Nakayama algebra with quiver of type $A_{n}$ for any natural number $n \geq 2$. The method for this construction has been explained in [3]. In this paper we provide an explicit technique which uses almost split sequences of Nakayama algebra given in [4], [3], and also uses the relationship between Nakayama algebra of type $A_{n-1}$ with type $A_{n}$ to give the construction by induction.

Tilting theory is one of essential tools in the representation theory of algebra. Tilting theory firstly appeared in the study of reflection functors ([5-7]). Tilting theory is a method for constructing a new algebra $B$ from original algebra $A$ such that the module categories are "close" to each other. The method is to construct an $A$-module $T$, called a tilting module and set a new algebra $B=$ End $T_{A}$. In this paper, as an application of the main theorem, we will construct a

Received September $14^{\text {th }}, 2011,1^{\text {st }}$ Revision February $21^{\text {st }}, 2012,2^{\text {nd }}$ Revision May $16^{\text {th }}, 2012,3^{\text {rd }}$ Revision September $27^{\text {th }}, 2012$, Accepted for publication November $5^{\text {th }}, 2012$.

Copyright @ 2013 Published by ITB Journal Publisher, ISSN: 2337-5760, DOI: 10.5614/j.math.fund.sci.2013.45.1.1 
tilting module of Nakayama algebra with quiver type Dynkin graph $A_{n}$ for all natural number $\mathrm{n} \geq 2$.

\section{$2 \quad$ Auslander Reiten Quiver}

Throughout this paper, algebras are basic and connected finite dimensional algebras over a fixed algebraically closed field $K$. For an algebra $A$, we denote by $\bmod A$ the category of finitely generated right $A$-modules. An algebra $A$ is said to be representation finite if the number of the isomorphism classes of indecomposable right $A$-module is finite. A $K$-algebra $A$ is called representationinfinite if $A$ is not representation-finite. An $A$-homomorphism module is a section (or a retraction) whenever it admits a left inverse (or a right inverse, respectively).

To construct the AR-quiver of an algebra we need some tools. These tools are the notion of almost split sequences and irreducible morphisms. Before we introduce this theory we give some definitions and theorems from [4] and [2].

Definition 1. Let $L, M$ be modules in $\bmod A$. An $A$-module homomorphism

$f: L \rightarrow M$ is called left minimal almost split if

(a) every $h \in$ End $M$ such that $h f=f$ is an automorphism,

(b) $f$ is not section,

(c) for every $A$-homomorphism $u: L \rightarrow \mathrm{U}$ that is not section there exists $u^{\prime}: L \rightarrow U$ such that $u^{\prime} f=u$.

A right minimal almost split homomorphism is defined dually.

Definition 2. A homomorphism $f: X \rightarrow Y$ in $\bmod A$ is said to be irreducible if

(a) $f$ is neither a section nor a retraction and

(b) if $f=f_{1} f_{2}$, either $f_{1}$ is a retraction or $f_{2}$ is a section.

We may think an irreducible morphism as a component of a right (left) minimal almost split by the following theorem.

Theorem 1. (a) Let $f: L \rightarrow M$ be left minimal almost split in $\bmod A$. Then $f$ is irreducible. Moreover, a homomorphism $f^{\prime}: L \rightarrow M$ 'of $A$-modules is irreducible if and only if $M^{\prime} \neq 0$ and there exists a direct sum decomposition $M \cong M^{\prime} \oplus M^{\prime \prime}$ and a homomorphism $f^{\prime \prime}: L \rightarrow M^{\prime \prime}$ such that $\left[\begin{array}{l}f^{\prime} \\ f^{\prime \prime}\end{array}\right]: L \rightarrow M^{\prime} \oplus M^{\prime \prime}$ is left minimal almost split. (b) Let $g: M \rightarrow N$ be right minimal almost split. Then $g$ is irreducible. Moreover, a homomorphism $g^{\prime}: M^{\prime} \rightarrow N$ of $A$-modules is irreducible 
if and only if $M^{\prime} \neq 0$ and there exists decomposition $M \cong M^{\prime} \oplus M^{\prime \prime}$ and a homomorphism $g^{\prime \prime}: M^{\prime \prime} \rightarrow N$ such that $\left[g^{\prime} g^{\prime \prime}\right]: M^{\prime} \oplus M^{\prime \prime} \rightarrow N$ is right minimal almost split.

Proof. See [2, section IV.1].

Theorem 2. (a) Let $S$ be a simple projective noninjective module in mod $A$. If $f: S \rightarrow M$, then $M$ is projective. (b) Let $S$ be a simple injective nonprojective module in $\bmod A$. If $g: M \rightarrow S$ is irreducible, then $M$ is injective.

Proof. See [2, section IV.3].

Let $X$ and $Y$ be modules in $\bmod A$, define $\operatorname{rad}_{A}(X, Y)=\left\{h \in \operatorname{Hom}(X, Y): 1_{X}-g h\right.$ is invertible for any $g \in \operatorname{Hom}(Y, X)\}$.

We define $\operatorname{rad}_{A}^{2}(X, Y)$ to consist of all $A$-module homomorphism of the form $g f$, where $f \in \operatorname{rad}_{A}(X, Z)$ and $g \in \operatorname{rad}_{A}(Z, Y)$.

Definition 3. A short exact sequence in $\bmod A$

$$
0 \rightarrow L \stackrel{f}{\rightarrow} M \stackrel{g}{\rightarrow} N \rightarrow 0
$$

is called an almost split sequence if $f$ is left minimal almost split.

We have several equivalent characterization of almost split sequences.

Theorem 3. Let $0 \rightarrow L \stackrel{f}{\rightarrow} M \stackrel{g}{\rightarrow} N \rightarrow 0$ be a short exact sequence in $\bmod A$. The following assertions are equivalent:

(a) The given sequence is almost split.

(b) $f$ is left minimal almost split.

(c) $g$ is right minimal almost split

(d) $L$ and $N$ are indecomposable, and $f$ and $g$ are irreducible.

Proof. See [2, section IV.1].

The following propositions are used to get the right (left) minimal almost split ending (or starting) at an indecomposable projective (or injective, respectively) module.

Proposition 1. (a) Let $P$ be an indecomposable projective module in $\bmod A$. An $A$-module homomorphism $g: M \rightarrow P$ is right minimal almost split if and only if $g$ is a monomorphism with image equal to $\operatorname{rad} P$. (b) Let $I$ be an 
indecomposable injective module in $\bmod A$. An $A$-module homomorphism $f: I$ $\rightarrow M$ is left minimal almost split if and only if $f$ is an epimorphism with kernel equal to $\operatorname{soc} I$.

Proof. See [2, section IV.3].

Let $M$ and $N$ be indecomposable modules in $\bmod A$, then $\operatorname{rad}_{A}(M, N)$ is the $K$ vector space of all noninvertible homomorphism from $M$ to $N$. Denote $\operatorname{Irr}(M, N)$ $=\operatorname{rad}_{A}(M, N) / \operatorname{rad}_{A}^{2}(M, N)$, that is the quotient of the $K$-vector spaces $\operatorname{rad}_{A}(M, N)$ and $\operatorname{rad}_{A}^{2}(M, N)$.

Definition 4. Let $A$ be a basic and connected finite dimensional $K$-algebra. The Auslander Reiten-quiver $\Gamma(\bmod A)$ of $\bmod A$ is defined as follows:

(a) The points of $\Gamma(\bmod A)$ are the isomorphism classes $[X]$ of indecomposable modules $X$ in $\bmod A$.

(b) Let $[M],[N]$ be the points in $\Gamma(\bmod A)$ corresponding to the indecomposable modules $M, N$ in $\bmod A$. The arrows $[M] \rightarrow[N]$ are in bijective correspondence with the vectors of a basis of the $K$-vector space $\operatorname{Irr}(M, N)$.

One of properties of the AR-quiver of representation finite algebra is given by the following proposition.

Proposition 2. Let $A$ be a representation-finite algebra. Then $\Gamma(\bmod A)$ has no multiple arrows.

Proof. See [2, section IV.4].

Now we recall a module which can be thought of as being close to the Morita progenerator.

Definition 5. A module $T$ in $\bmod A$ is called a tilting module if the following three conditions are satisfied:

(T1) the projective dimension of $T$ is at most one,

(T2) $\operatorname{Ext}_{A}^{1}(T, T)=0$,

(T3) there exists a short exact sequence $0 \rightarrow A_{A} \rightarrow T_{A}^{\prime} \rightarrow T^{\prime \prime}{ }_{A} \rightarrow 0$ with $T^{\prime}, T^{\prime \prime}$ $\in$ add $T$.

Note that any Morita progenerator is a tilting module and any projective $A$ module always satisfy both (T1) and (T2). It is easy to see that every tilting module is faithful. 


\section{The Construction of Nakayama algebra type Dynkin Graph $A_{n}$}

Now we will describe the AR-quiver of a Nakayama algebra $A$ with a quiver of type $A_{n}$. An algebra $A$ is called Nakayama algebra if it is both right serial and left serial. For a basic and connected algebra there are only two types of Nakayama algebra.

Theorem 4. A basic and connected algebra $A$ is a Nakayama algebra if and only if its quiver representation $Q_{A}$ is one of the following two quivers:

(a)

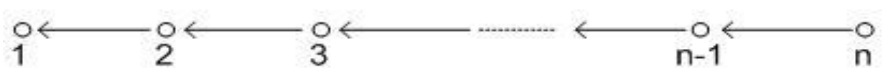

(b)

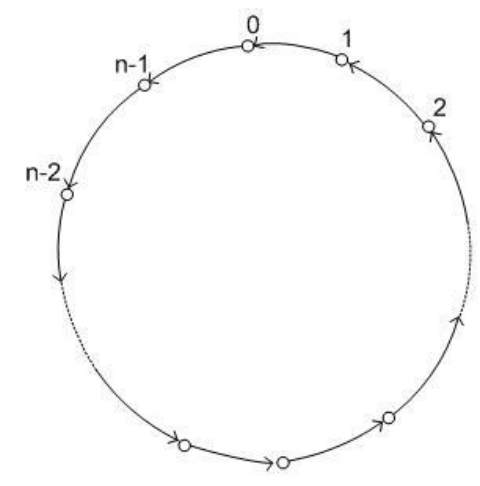

(with $n \geq 1$ points).

Proof. See [2, section V.3].

The first Nakayama algebra will be called the Nakayama algebra type Dynkin graph $A_{n}$. The following theorem and corollary are some properties of Nakayama algebras.

Theorem 5. Let $A$ be a basic and connected Nakayama algebra, and let $M$ be an indecomposable $A$-module. There exists an indecomposable projective $A$ module $P$ and an integer $t$ with $1 \leq t \leq \ell \ell(P)$ where $\ell \ell(P)$ is the Loewy length of $P$, such that $M \cong P / \operatorname{rad}^{t} P$. In particular, $A$ is representation finite.

Proof. See [2, section V.3].

Corollary 1. A basic and connected algebra $A$ is a Nakayama algebra if and only if every indecomposable $A$-module is uniserial. 
Proof. See [2, section V.3].

Before constructing the AR-quiver, we will need the following result. Let $Q$ be the quiver

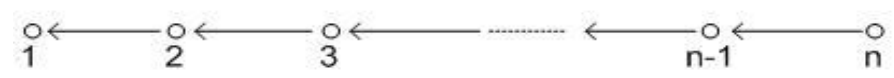

and $Q^{\prime}$ be the quiver

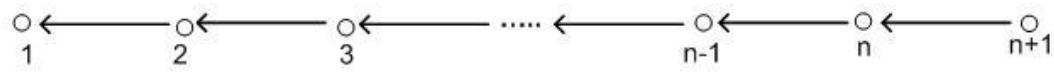

Write $Q_{0}=\{1,2, \ldots, n\}, Q^{\prime}=\{1,2, \ldots, n+1\}, Q_{1}=\left\{\alpha_{i}: i+1 \rightarrow i \mid i \in Q_{0} \backslash\{n\}\right\}$ and $Q^{\prime}{ }_{1}=Q_{1} \cup\left\{\alpha_{n}: n+1 \rightarrow n\right\}$. Set $A=K Q$ and $A^{\prime}=K Q^{\prime}$.

Lemma 1. Let $M=\left(M_{i}, \varphi_{\alpha}\right)_{i \in Q_{0}, \alpha \in Q_{1}}$ be a module in $\bmod A$. Define $M=$

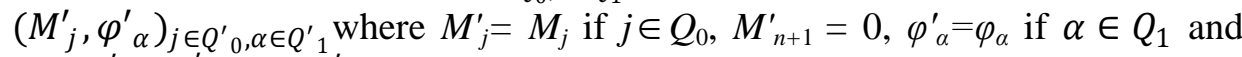
$0=\varphi_{\alpha_{n}}^{\prime}: M_{n+1}^{\prime} \rightarrow M_{n}^{\prime}$. The $A$-module $M$ is indecomposable if and only if the $A^{\prime}$ '-module $M^{\prime}$ ' is indecomposable.

Proof. $(\Rightarrow)$ Assume that $M$ is indecomposable., Suppose that $M^{\prime}$ is

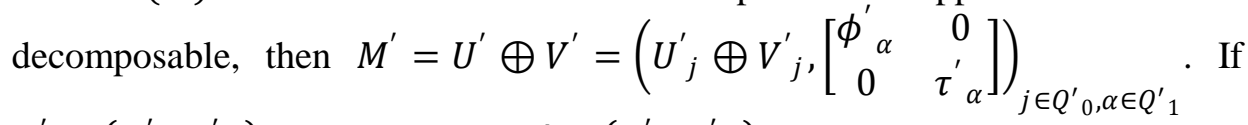
$U^{\prime}=\left(U_{j}^{\prime}, \phi_{\alpha}^{\prime}\right)_{j \in Q_{0}^{\prime}, \alpha \in Q^{\prime}{ }_{1}}$ and $V^{\prime}=\left(V_{j}^{\prime}, \phi_{\alpha}^{\prime}\right)_{j \in Q_{0}^{\prime}, \alpha \in Q^{\prime}{ }_{1}}$ then $U_{n+1}^{\prime}=0=V_{n+1}^{\prime}$. Set $U=\left(U_{i}, \phi_{\alpha}\right)_{i \in Q_{0}, \alpha \in Q_{1}}$ and $V=\left(V_{i}, \tau_{\alpha}\right)_{i \in Q_{0}, \alpha \in Q_{1}}$ where $U_{i}=U_{i}^{\prime}, V_{i}=V_{i}^{\prime}$ and $\phi_{\alpha}=\phi_{\alpha}^{\prime}, \tau_{\alpha}=\tau_{\alpha}^{\prime}$. By definition, we have $M=K \oplus L$. This contradiction shows that $M^{\prime}$ is indecomposable.

$(\Leftarrow)$ Assume that $M^{\prime}$ is indecomposable. Suppose that $M$ is decomposable.We have $M=U \oplus V=\left(U_{i} \oplus V_{i},\left[\begin{array}{cc}\phi_{\alpha} & 0 \\ 0 & \tau_{\alpha}\end{array}\right]\right)_{i \in Q_{0}, \alpha \in Q_{1}}$ with $U$ and $V$ are nonzero submodules of $M$. By definition, $M^{\prime}=U^{\prime} \oplus V^{\prime}$ where $U^{\prime}=\left(U_{j}^{\prime}, \phi_{\alpha}^{\prime}\right)_{j \in Q^{\prime}{ }_{0}, \alpha \in Q^{\prime}{ }_{1}}$ and $V^{\prime}=\left(V_{j}^{\prime}, \tau_{\alpha}^{\prime}\right)_{j \in Q_{0}^{\prime}, \alpha \in Q^{\prime}{ }_{1}}$ with $U_{j}=U_{j}^{\prime}, V_{j}=V_{j}^{\prime}$ if $j \in Q_{0}, U_{n+1}^{\prime}=0=V_{n+1}^{\prime}$ and $\phi_{\alpha}^{\prime}=\phi_{\alpha}, \tau_{\alpha}^{\prime}=\tau_{\alpha}$ if $\alpha \in Q_{1}, \phi_{\alpha_{n}}^{\prime}=0, \tau_{\alpha_{n}}^{\prime}=0$. This contradict that $M^{\prime}$ is indecomposable.

Lemma 2. Let $M$ and $M^{\prime}$ be the modules in Lemma 1 . The $A$-module $M$ is simple if and only if the $A^{\prime}$-module $M^{\prime}$ is simple. 
Proof. Note that any simple both $A$-module and $A^{\prime}$-module are 1-dimensional. It follows from the total dimension of $K$-vector space in linear representation of $M$ and $M^{\prime}$, we have $\operatorname{dim}_{K}(M)=1$ if and only if $\operatorname{dim}_{K}\left(M^{\prime}\right)=1$.

Both Lemma 1 and Lemma 2 above say that there exists an inclusion between the collection of isomorphism classes of indecomposable (or simple) $A$-module and the collection of isomorphism classes of indecomposable (or simple) $A^{\prime}$ module.

Lemma 3. Let $M=\left(M_{i}, \varphi_{\alpha}\right)_{i \in Q_{0}, \alpha \in Q_{1}}, N=\left(N_{i}, \tau_{\alpha}\right)_{i \in Q_{0}, \alpha \in Q_{1}}$ be modules in $\bmod A$ and let $M^{\prime}, N^{\prime}$ be modules defined in the same way like in Lemma 1. Let $f=\left(f_{i}\right)_{i \in Q_{0}}: M \rightarrow N$ and $f^{\prime}=\left(f^{\prime}{ }_{j}\right)_{j \in Q^{\prime}{ }_{0}}: M^{\prime} \rightarrow N^{\prime}$ where $f_{j}^{\prime}=f_{j}$ if $j \in Q_{0}$ and $f_{n+1}^{\prime}=0: M_{n+1}^{\prime} \rightarrow N_{n+1}^{\prime}$. If $f$ is an irreducible morphism then so is $f^{\prime}$.

Proof. It is clear that $f^{\prime}=\left(f^{\prime}{ }_{j}\right)_{j \in Q_{0}^{\prime}}$ is a morphism: indeed $f_{k}^{\prime} \varphi_{\alpha_{k}}^{\prime}=\tau_{\alpha_{k}}^{\prime} f_{k+1}^{\prime}$ for every $k \in Q_{0}$. We claim that $f$ has no both right inverse and left inverse. Suppose that $f^{\prime}$ has a right inverse $g^{\prime}=\left(g_{i}\right)_{i \in Q_{0}^{\prime}}$. It means that $f_{i}^{\prime} g_{i}=1_{N_{i}^{\prime}}$ for every $i \in\{1,2, \ldots, n+1\}$. Consequently, if $i \in Q_{0}$ then $f_{i} g_{i}=f_{i}^{\prime} g_{i}=1_{N_{i}^{\prime}}=1_{N_{i}}$. Define $g=\left(g_{i}\right)_{i \in Q_{0}}$, then $g$ is a morphism in $\bmod A$, moreover $f g=1_{N}$, contrary to our assumption. So $f$ has no right inverse. In a similar way, we conclude that $f$ has no left inverse also, claim proved.

Now, let $f^{\prime}=h^{\prime} g^{\prime}$ and assume that $h^{\prime}$ is not a retraction. If $h^{\prime}=\left(h_{1}, h_{2}, \ldots, h_{n}, h_{n+1}\right)$ and $g^{\prime}=\left(g_{1}, g_{2}, \ldots, g_{n}, g_{n+1}\right)$ then $h_{n+1}=0$ and $g_{n+1}=0$. We get $f=h g$ where $h=\left(h_{l}\right.$, $\left.h_{2}, \ldots, h_{n}\right)$ and $g^{\prime}=\left(g_{1}, g_{2}, \ldots, g_{\mathrm{n}}\right)$. Because $h^{\prime}$ is not a retraction, then $h$ is not a retraction. This means that $g$ is a section because $f$ is irreducible. Hence, $g^{\prime}$ is a section. This proves that $f$ is irreducible.

Now let $2 \leq n \in \mathbb{N}$ and $A$ be a Nakayama algebra of type $A_{n}$. To start to build the AR-quiver of $A$ we first list all isomorphism classes of indecomposable projective $A$-module, indecomposable injective and simple. Each of modules will be considered as linear representation and the vertices were replaced by the dimension of its vector space. All indecomposable projective modules of $A$ are:

$$
\begin{aligned}
& P(1)=\underbrace{100 \ldots 00}_{n} \\
& P(2)=110 \ldots 00 \\
& P(3)=111 \ldots 00
\end{aligned}
$$




$$
\begin{gathered}
P(i)=\underbrace{11 \ldots 1}_{i} 0 \ldots 00 \\
\vdots \\
P(n-1)=111 \ldots 10 \\
P(n)=111 \ldots 11
\end{gathered}
$$

All indecomposable injective modules of $A$ are:

$$
\begin{gathered}
I(1)=\underbrace{111 \ldots 11}_{n} \\
I(2)=011 \ldots 11 \\
I(3)=001 \ldots 11 \\
\vdots \\
I(i)=\underbrace{00}_{\begin{array}{c}
0.1-1 \\
\vdots
\end{array}} 1 \ldots 11 \\
I(n-1)=000 \ldots 011 \\
I(n)=000 \ldots 01
\end{gathered}
$$

and all simple modules are:

$$
\begin{aligned}
& S(1)=\underbrace{100 \ldots 00}_{n} \\
& S(2)=010 \ldots 00 \\
& S(3)=001 \ldots 00 \\
& S(i)=\underset{i-t h \text { position }}{000 \ldots 01000} \\
& S(n-1)=000 \ldots 10 \\
& S(n)=000 \ldots 01
\end{aligned}
$$

We have $P(1)=S(1), P(n)=I(1), I(n)=S(n)$. Further, $I(k)=\frac{P(n)}{P(k-1)}$ for every $k \in\{1,2, \ldots, n\}$ with $P(0)=0$. It easy to check that $\operatorname{rad} P(k)=P(k-1)$ for every $k$ $\in\{1,2, \ldots, n\}$. In other words $\operatorname{rad}^{t} P(n)=P(n-t)$, with $t \leq n$. By Proposition 1 , the inclusion $i_{j}: P(j-1)=\operatorname{rad} P(j) \rightarrow P(j)$ is a right minimal almost split for every $j \in\{2,3, \ldots, n\}$ and the canonical homomorphism $p_{k}: I(k) \rightarrow I(k+1)=$ $\frac{I(k)}{\operatorname{soc} I(k)}$ is a left minimal almost split for every $k \in\{1,2, \ldots, n-1\}$.

Lemma 4. (a) The sequence $0 \rightarrow P(1) \stackrel{i}{\rightarrow} P(2) \stackrel{p}{\rightarrow} S(2) \rightarrow 0$ is almost split, where $i$ is the inclusion and $p$ is the canonical homomorphism with kernel equal 
to $P(1)$.(b) The sequence $0 \rightarrow S(n-1) \stackrel{i^{\prime}}{\rightarrow} I(n-1) \stackrel{p^{\prime}}{\rightarrow} S(n) \rightarrow 0$ is almost split, where $i^{\prime}$ is the inclusion and $p^{\prime}$ is the canonical homomorphism with kernel equal to $S(n-1)$.

Proof. (a) It is clear that the sequence in (a) is exact. Because $P(2)=\operatorname{rad} P(1)$, then $i: P(1) \rightarrow P(2)$ is a right minimal almost split (by Proposition 1(a)). We know that $P(1)$ is indecomposable, using Theorem 1(b), the inclusion $i$ is the only irreducible morphism ending with $P(2)$. Let $h: P(1) \rightarrow M$ be a left minimal almost split. Because $P(1)$ is a simple projective noninjective module in $\bmod A$, then by Theorem $2, M$ is a projective module. Without loss of generality, by $t$ Proposition 2 we may assume that $M=\bigoplus_{j=1} P(j)$ with the $P(j)$ indecomposa-

ble projective and pairwise nonisomorphic. This means that $h_{j}: P(1) \rightarrow P(j)$ is irreducible and consequently $j=1$ and $M=P(j)$. We have a right minimal almost split $g$ : $\operatorname{rad} P(j) \rightarrow P(j)$. This means that $P(1)$ is a direct summand of rad $P(j)$. But $\operatorname{rad} P(j)=P(j-1)$ is indecomposable, it follows that $M=P(2)$. Thus, $h$ $=i: P(1) \rightarrow P(2)$ is a left minimal almost split. By Theorem 3(b), the given sequence is almost split, we have proved part (a).

(b) It is clear that the sequence in (b) is exact. We know that the canonical homomorphism $s: I(n-1) \rightarrow S(n)=I(n)$ is left minimal almost split. Since $S(n)$ is indecomposable, using Theorem 1(a), the inclusion $i$ ' is the only irreducible morphism starting with $I(n-1)$. Let $f: M \rightarrow I(n)$ be right minimal almost split. Since $S(n)=I(n)$ is a simple injective nonprojective, by Theorem $2, M$ is injective. Without loss of generality, by Proposition 2 we may assume that $M=\underset{j=1}{\bigoplus} I(j)$ with the $I(j)$ indecomposable injective and pairwise nonisomorphic. Thus, $f_{j}: I(j) \rightarrow I(n)$ is irreducible and implies $t \leq n-1$. We have a left minimal almost split $I(j) \rightarrow I(j+1)=\frac{I(j)}{\operatorname{soc} I(j)}$ This means that $I(n)$ is direct summand of $I(j+1)$. Since $I(j+1)$ is indecomposable, then $M=I(n)$. Hence, $f=p: I(n-1) \rightarrow I(n)$ is a right minimal almost split. It follows from Theorem 3(c) this sequence is almost split.

Lemma 5. Let $2 \leq n \in \mathbb{N}$ and $A=K Q$ where $Q$ is a quiver of Dynkin type $A_{n}$. Then for every $j \in\{1,2, \ldots, n-1\}$, the sequence $0 \rightarrow S(j) \stackrel{i}{\rightarrow} V_{j, j+1} \stackrel{p}{\rightarrow} S(j+$ 1) $\rightarrow 0$ is almost split where $V_{j, j+1}=00 \ldots 0 \underbrace{11}_{j, j+1} 0 \ldots 00$. 
Proof. We proceed by induction on $n$. Let $n=2,3$, it follows from Lemma 4 that this sequence is almost split. Now assume that $\mathrm{n}>4$, if $j=1$ and $j=n-1$ then the proof follows from Lemma 4 . If $j \in\{2, \ldots, n-2\}$, it is clear the sequence $0 \rightarrow S(j) \stackrel{i}{\rightarrow} V_{j, j+1} \stackrel{p}{\rightarrow} S(j+1) \rightarrow 0$ is exact. By hypothesis the sequence $0 \rightarrow S(j) \stackrel{i}{\rightarrow} V_{j, j+1} \stackrel{p}{\rightarrow} S(j+1) \rightarrow 0$ in $\bmod B=K Q^{\prime}$ with $Q^{\prime}$ is quiver type $A_{n-1}$ is almost split. In other words, $i$ and $p$ are irreducible morphism in $\bmod B$. Now consider the exact sequence $0 \rightarrow S^{\prime}(j) \stackrel{i^{\prime}}{\rightarrow} V_{j, j+1}^{\prime} \stackrel{p^{\prime}}{\rightarrow} S^{\prime}(j+1) \rightarrow 0 \operatorname{in} \bmod A$. By Lemma 3, $i^{\prime}$ and $p^{\prime}$ are irreducible morphisms in $\bmod A$. It follows from Theorem 3 (d) that the sequence $0 \rightarrow S^{\prime}(j) \stackrel{i^{\prime}}{\rightarrow} V_{j, j+1}^{\prime} \stackrel{p^{\prime}}{\rightarrow} S^{\prime}(j+1) \rightarrow 0$ is almost split, the lemma is proved.

Proposition 3. Let $P$ be a nonsimple indecomposable projective-injective module, $S=\operatorname{soc} P$ and $R=\operatorname{rad} P$. Then the sequence

$$
0 \rightarrow R \stackrel{\left[\begin{array}{l}
q \\
i
\end{array}\right]_{R}}{\rightarrow} \oplus P \stackrel{[-j \quad p}{\longrightarrow} P / S \rightarrow 0
$$

is almost split, where $i, j$ are the inclusion and $p, q$ the projections.

Proof. See [2, section IV.3].

Corollary 2. The sequence

$$
0 \rightarrow P(n-1) \stackrel{\left[\begin{array}{l}
q \\
\rightarrow
\end{array}\right]}{\longrightarrow} \frac{P(n-1)}{P(1)} \oplus P(n) \stackrel{[-j \quad p]}{\longrightarrow} P(n) / P(1) \rightarrow 0
$$

is almost split in $\bmod A$.

Proof. Apply Proposition 3.

Lemma 6. The sequence

$$
0 \rightarrow \frac{P(n-1)}{P(k)} \stackrel{\left[\begin{array}{l}
q \\
i
\end{array}\right]}{\longrightarrow} \frac{P(n-1)}{P(k+1)} \oplus \frac{P(n)}{P(k)} \stackrel{[-j \quad p]}{\longrightarrow} P(n) / P(k+1) \rightarrow 0
$$

is almost split in $\bmod A$, where $k \in\{1,2, \ldots, n-3\}$.

Proof. It is clear that the above sequence is exact. Because $\frac{P(n-1)}{P(k)}, \frac{P(n-1)}{P(k+1)}, \frac{P(n)}{P(k)}$, $P(n) / P(k+1)$ are indecomposable and pairwise nonisomorphic, this sequence is not split. Using Theorem 3, it suffices to show that the homomorphism $g=[-j p]$ is right almost split. Clearly $g$ is not a retraction. Let $V$ be an indecomposable $A$ module, $M=\frac{P(n)}{P(k+1)}$ and $v: V \rightarrow M$ is not an isomorphism. We have two cases. 
If $v$ is not surjective, then $\operatorname{Im}(v)$ is contained in the unique maximal submodule (because $M$ is uniserial) $\operatorname{rad} M$ of $M$. We compute $\operatorname{rad} M$ by its linear representation, then we have $\operatorname{rad} M=\frac{P(n-1)}{P(k+1)}$. Hence, we get a homomorphism $\left[\begin{array}{c}-v \\ 0\end{array}\right]: V \rightarrow \frac{P(n-1)}{P(k+1)} \oplus \frac{P(n)}{P(k)}$ that satisfies $g\left[\begin{array}{c}-v \\ 0\end{array}\right]=v$. If $v$ is surjective, then $\frac{V}{\operatorname{Ker} V} \cong M=\frac{P(n)}{P(k+1)}$. Since $\frac{V}{\operatorname{Ker} V}$ is uniserial and $v$ is not an isomorphism, Ker $v$ $=\operatorname{rad}^{s} V$ with $s \geq 1$ and and $\operatorname{dim}_{K}(V)>n-(k+1)$. Since $V$ is indecomposable, by Theorem 5 we get $V \cong M=\frac{P(m)}{\operatorname{rad}^{r} P(m)}=\frac{P(m)}{P(m-r)}$ with $1 \leq m \leq n$ and $1 \leq r \leq m$, thus $\mathrm{r}=\operatorname{dim}_{K}(V)>n-(k+1)$. We have $\operatorname{rad}^{\mathrm{s}} V=\operatorname{rad}^{\mathrm{s}}\left(\frac{P(m)}{P(m-r)}\right)=\frac{\operatorname{rad}^{s} P(m)}{P(m-r)}=$ $\frac{P(m-s)}{P(m-r)}$. Consequently, $\frac{V}{\operatorname{Ker} V}=\frac{P(m) / P(m-r)}{P(m-s) / P(m-r)}$. Using its linear representation we get $m=n$ : indeed $\frac{V}{\operatorname{Ker} V}$ is indecomposable injective. Hence, $V=\frac{P(n)}{P(n-r)}$ with $n$ $r<k+1$. Thus, there exists an epimorphism $v^{\prime}: V \rightarrow \frac{P(n)}{P(r)}$ such that $v=p v^{\prime}$. Define a homomorphism $\left[\begin{array}{c}0 \\ v^{\prime}\end{array}\right]: V \rightarrow \frac{P(n-1)}{P(k+1)} \oplus \frac{P(n)}{P(k)}$, then $g \cdot\left[\begin{array}{c}0 \\ v^{\prime}\end{array}\right]=v$. Assume now that $f: N \rightarrow M$ is not a retraction and let $N=\bigoplus N(k)$, then for every $k$, $N_{k} ¥ M$. As we have seen before there exists $h_{k}: N_{k} \rightarrow \frac{P(n-1)}{P(k+1)} \oplus \frac{P(n)}{P(k)}$ such that $g\left[h_{1} h_{2} \ldots h_{t}\right]=\left[f_{1} f_{2} \ldots f_{t}\right]$ where $f_{i}: N_{i} \rightarrow M$ is a component of $f$. We conclude that $g$ is right almost split and thus the given sequence is almost split.

In the process of proving the main result we need one fact about the AR-quiver with connected components.

Theorem 6. Assume that $A$ is a basic and connected finite dimensional $K$ algebra. If $\Gamma(\bmod A)$ admits a connected component $C$ whose modules are of bounded length, then $C$ is finite and $\mathrm{C}=\Gamma(\bmod A)$. In particular, $A$ is representation finite.

Proof. See [2, section IV.5].

Now we give the main results in this paper

Theorem 7. Let $Q$ be a quiver of type $A_{n}$ with $n \in \mathbb{N}$ and $\mathrm{n} \geq 2$. Let $A=K Q$, then $\Gamma(\bmod A)$ is the quiver 


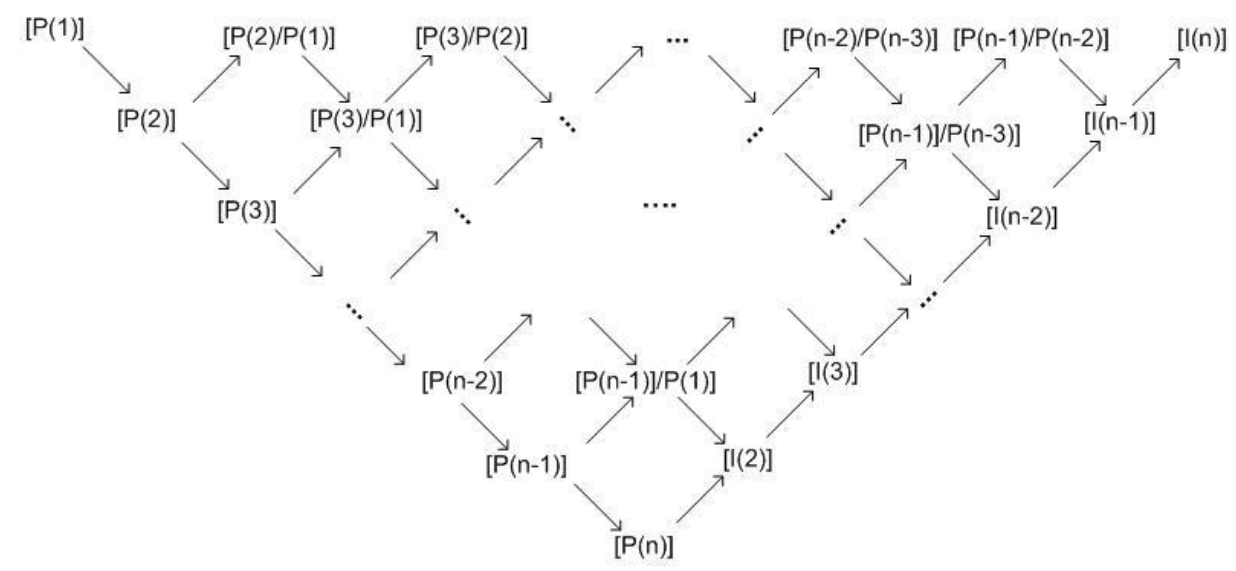

Proof. We proceed by induction on $n$. For $n=2,3$ it is easy to check that its ARquiver satisfy the given picture. Before we continue our induction process, we need the following useful fact: every subquiver of $\Gamma(\bmod A)$ of the form smallest diamond with 4 arrows, its vertices form an almost split sequence. Precisely, if we take a subquiver of $\Gamma(\bmod A)$ of the form

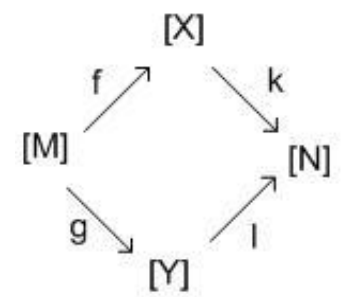

with $f: M \rightarrow X, g: M \rightarrow Y, k: X \rightarrow N, l: Y \rightarrow N$ are irreducible morphism then the sequence

$$
0 \rightarrow M \stackrel{s=\left[\begin{array}{l}
f \\
g
\end{array}\right]}{\longrightarrow} X \oplus Y \stackrel{t=\left[\begin{array}{ll}
-k & l
\end{array}\right]}{\longrightarrow} N \rightarrow 0
$$

is almost split. It can be checked that $f, l$ are projection and $g, k$ are inclusion. Note that this fact holds for $n=2,3$. Now assume that the statement is true for $n$-1 and denote $A^{\prime}=K Q^{\prime}$ with $Q^{\prime}$ quiver of Nakayama algebra type $A_{n-1}$. It follows that its AR-quiver is 


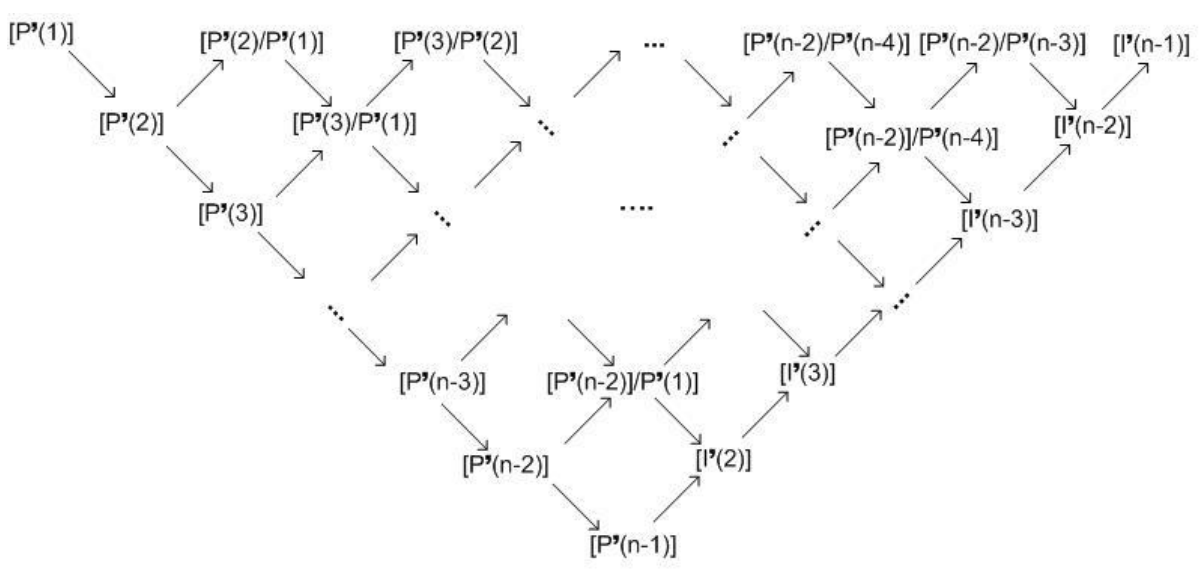

and for every smallest diamond form an almost split sequence as we say before. By Lemma 1 we get some isomorphism classes of indecomposable A-module such that the number of its is the same as the number of vertices of $\Gamma\left(\bmod A^{\prime}\right)$. By the induction hypothesis and Lemma 3 , we get a subquiver of $\Gamma(\bmod A)$ :

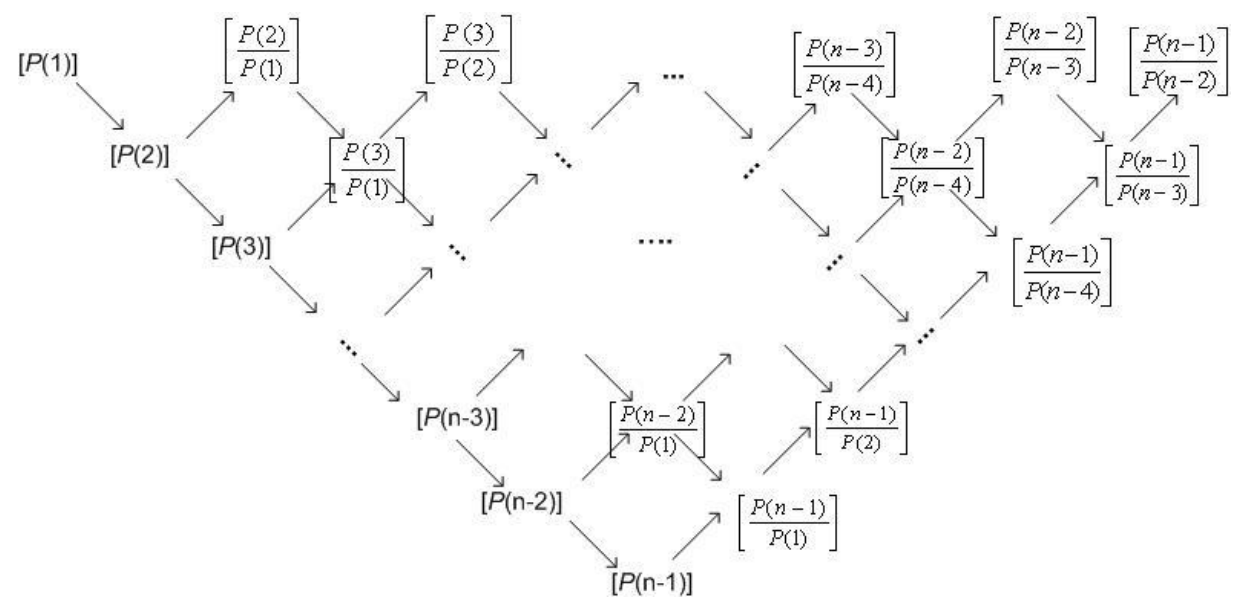

We claim that for every subquiver of the form smallest diamond in this quiver form an almost split sequence. We have the sequence

$$
0 \rightarrow M \stackrel{s=\left[\begin{array}{l}
f \\
g
\end{array}\right]}{\longrightarrow} X \oplus Y \stackrel{t=\left[\begin{array}{ll}
-k & l
\end{array}\right]}{\longrightarrow} N \rightarrow 0
$$

by taking the smallest diamond subquiver $\Gamma\left(\bmod A^{\prime}\right)$. Let $M^{\prime}, N^{\prime}, X^{\prime}, Y^{\prime}$ be $A$ modules defined as same as modules in Lemma 1. Thus, we have an exact sequence 


$$
0 \rightarrow M^{\prime} \stackrel{s^{\prime}=\left[\begin{array}{l}
f^{\prime} \\
g^{\prime}
\end{array}\right]}{\longrightarrow} X^{\prime} \oplus Y^{\prime} \stackrel{t^{\prime}=\left[\begin{array}{ll}
-k^{\prime} & l^{\prime}
\end{array}\right]}{\longrightarrow} N^{\prime} \rightarrow 0
$$

where $f^{\prime}, l^{\prime}$ are projection and $g^{\prime}, k^{\prime}$ are inclusion. Because $s, t$ are irreducible morphisms, then by Lemma 3 we have that $s^{\prime}, t^{\prime}$ are irreducible morphisms in $\bmod A$. It follows from Theorem 3 the latter exact sequence is almost split, and our claim proved. By Corollary 2, Lemma 5 and Lemma 6 we get a connected component of $\Gamma(\bmod A)$ :

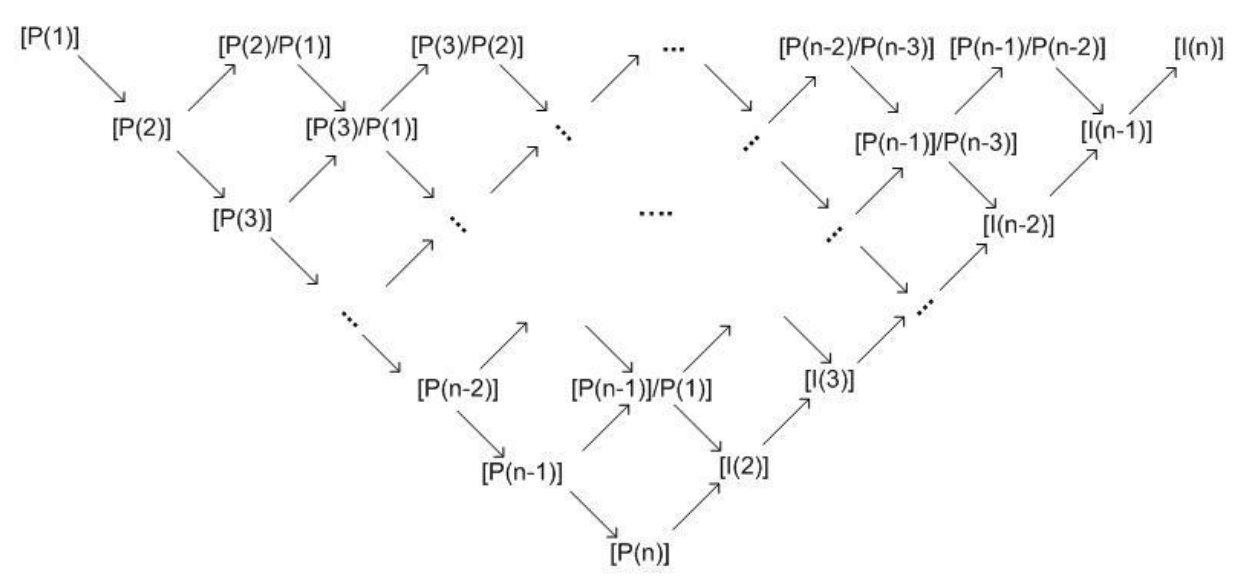

It follows from Theorem 5 the connected component is same as with the $\Gamma(\mathrm{mod}$ A). This completes the proof.

Corollary 3. Let $A$ be a Nakayama algebra with quiver type $A_{n}$. The number of the isomorphism classes of indecomposable A-module is $\frac{1}{2} n(n+1)$.

Proof. We proceed by induction on $n$. For $n=2,3$ we can directly compute from its quiver and we get both the number of the isomorphism classes of indecomposable modules are 3 and 6 respectively. Now assume that for quiver type $A_{n-1}$ the number of the isomorphism classes of indecomposable modules is $\frac{1}{2}(n-1) n$. We have seen that in the proof of our main theorem, $\Gamma(\bmod A)$ has a subquiver which isomorphic to AR-quiver of Nakayama algebra type $A_{n-1}$. Further, all indecomposable modules in this subquiver are noninjective. This means, the number of the isomorphism classes indecomposable noninjective of A-module is $\frac{1}{2}(n-1) n$. But, we know that $A$ has $n$ isomorphism classes of indecomposable injective $A$-modules. Hence, the number of the isomorphism classes of indecomposable $A$-modules is $\frac{1}{2}(n-1) n+n=\left(\frac{1}{2}(n-1)+1\right) n=$ 
$\frac{1}{2} n(n+1)$

AR-quiver has an important role in studying the category of modules. It stores the information of indecomposable modules, homomorfisms between indecomposable modules and almost split sequences. Now by using AR-quiver constructed above we may give a tilting module of algebra $A=K Q$ with $Q$ is quiver of type $A_{n}$. Let $T=I(1) \oplus P(1) \oplus I(3) \oplus I(4) \oplus \ldots \oplus I(\mathrm{n})$, we claim that $T$ is a tilting module. Because $A$ is hereditary we have $\operatorname{gl} \operatorname{dim} A=1$. So projective dimension of $T$ is at most 1 . Since $T$ is not projective the projective dimension is equal 1. Let $M=I(3) \oplus \ldots \oplus I(n)$. Because $I(1) \oplus P(1)$ is projective, whereas $I(1) \oplus I(3) \oplus \ldots \oplus I(n)$ is injective, we have

$$
\begin{aligned}
\operatorname{Ext}_{A}^{1}(T, T) & \cong \operatorname{Ext}_{A}^{1}(M, P(1)) \cong D \operatorname{Hom}_{A}(P(1), \tau(M)) \\
& \cong D \operatorname{Hom}_{A}(P(1), \tau(I(3)) \oplus \ldots \oplus \tau(I(n))) \\
& \cong D \operatorname{Hom}_{\mathrm{A}}\left(\mathrm{P}(1), \frac{\mathrm{P}(\mathrm{n}-1)}{\mathrm{P}(1)} \oplus \ldots \oplus \frac{\mathrm{P}(\mathrm{n}-1)}{\mathrm{P}(\mathrm{n}-2)}\right) \\
& \cong D \operatorname{Hom}_{\mathrm{A}}\left(\mathrm{P}(1), \frac{\mathrm{P}(\mathrm{n}-1)}{\mathrm{P}(1)}\right) \oplus \ldots \oplus D \operatorname{Hom}_{\mathrm{A}}\left(\mathrm{P}(1), \frac{\mathrm{P}(\mathrm{n}-1)}{\mathrm{P}(\mathrm{n}-2)}\right)=0
\end{aligned}
$$

Note that, we have $\tau(\mathrm{I}(\mathrm{j}))=\frac{P(n-1)}{j-2}$, by looking its AR-quiver. Indeed, in [1] we know that for any indecomposable nonprojective $A$-module $M$, there exists an almost split sequence $0 \rightarrow \tau(M) \rightarrow E \rightarrow M \rightarrow 0$. This almost split sequence appears in AR-quiver in the form of a smallest diamond. To show $T$ satisfy (T3) it suffices to prove that for any indecomposable projective $A$-module $P$, there exists a short exact sequence

$$
0 \rightarrow P_{A} \rightarrow T_{A}^{\prime} \rightarrow T_{A}^{\prime \prime} \rightarrow 0 .
$$

with $T^{\prime}, T^{\prime \prime} \in$ add $T$. Because $P(1), P(n)=I(1) \in$ add $T$ we have short exact sequences

$$
\begin{aligned}
& 0 \rightarrow P(1) \rightarrow P(1) \oplus P(n) \rightarrow P(n) \rightarrow 0, \\
& 0 \rightarrow P(n) \rightarrow P(1) \oplus P(n) \rightarrow P(1) \rightarrow 0 .
\end{aligned}
$$

with $P(1) \oplus P(n) \in$ add $T$. On the other hand, for every $i$ with $2 \leq i \leq n-1$ there exists a short exact sequence

$$
0 \rightarrow P(i) \stackrel{f_{i}}{\rightarrow} P(n) \stackrel{g_{i}}{\rightarrow} I(i+1) \rightarrow 0,
$$

where $f_{i}$ is the inclusion and $g_{i}$ is the projection for all $i$. We conclude that $T$ is a tilting module of $A$. By the above reason we get the following result. 
Proposition 4. Let $A$ be the $K Q$ algebra with $Q$ is quiver of type $A_{n}$. Then $T=I(1) \oplus P(1) \oplus I(3) \oplus I(4) \oplus \ldots \oplus I(\mathrm{n})$ is a tilting module.

\section{Conclusion}

Our main result is a generalization of AR-quiver of the first kind Nakayama algebra. This AR-quiver have a nice structure and the isomorphism classes of its indecomposable module is easy to understand. In this AR-quiver the position of all simple modules is on the top of the AR-quiver. On the left side we have all indecomposable projective and on the right side we have all indecomposable injective. Since $A$ is a Nakayama algebra, all indecomposable module which not injective is only a quotient of indecomposable projective module. The ARquiver of any finite representation algebra has a role in finding all homomorphisms between indecomposable modules. By using this information, we get a tilting module of Nakayama algebra of type Dynkin graph $A_{n}$. We have that, if $T=I(1) \oplus P(1) \oplus I(3) \oplus I(4) \oplus \ldots \oplus I(\mathrm{n})$ then $T$ is a tilting module.

\section{Acknowledgement}

This research is supported by Hibah Riset dan Inovasi KK 2011 based on Surat Perjanjian no. 214 I.1.C01/PL/2011.

\section{References}

[1] Auslander, M., Representation Theory of Artin Algebras II, Comm. Algebra, 1, pp. 269-310, 1974.

[2] Auslander, M., Representation Theory of Artin Algebras III, Comm. Algebra, 3, pp. 269-310, 1975.

[3] Auslander, M., Reiten, I. \& Smalo, S.O., Representation Theory of Artin Algebras, Cambridge University Press, 1995.

[4] Assem, I., Simson, D. \& Skowronski, A., Elements of the Representation Theory of Associative Algebras, Cambridge University Press, 2006.

[5] Auslander, M., Platzeck, M.I. \& Reiten, I., Coxeter Functors without Diagrams, Trans. Amer. Math. Soc., 250, pp. 1-46, 1979.

[6] Bernstein, I.N., Gelfand, I.M. \& Ponomarev, V.A., Coxeter Functors and Gabriel's Theorem, Uspiehi Mat. Nauk, 28, pp. 19-33, 1973 (in Russian), English translation in Russian Math. Surveys, 28, pp. 17-32, 1973.

[7] Hugel, L.A., Happel, D. \& Krause, H., Handbook of Tilting Theory, Cambridge University Press, 2008. 